\title{
A New Representation of Efficient Point Sets and Its Applications in DEA
}

\author{
Maoqin Li \\ Department of Mathematics, Taizhou University, Linhai, Zhejiang 317000, China \\ Correspondence should be addressed to Maoqin Li; limaoqin@hotmail.com
}

Received 18 January 2013; Accepted 25 February 2013

Academic Editor: Zhuming Bi

Copyright (C) 2013 Maoqin Li. This is an open access article distributed under the Creative Commons Attribution License, which permits unrestricted use, distribution, and reproduction in any medium, provided the original work is properly cited.

$E(M, K)\left(E_{w}(M, K)\right)$, the set of Pareto efficient (weak efficient) points of a set $M$ with respect to a cone $K$ in $R^{n}$, is expressed as a differencebetween two sets $M$ and $M+K \backslash\{0\}(M$ and $M+\operatorname{int} K)$. Using the new representation, the properties of $E(M, K)$ are proved more easily than before. When $M$ or $K$ is in the form of union, intersection, sum, or difference of two sets or two cones, respectively, the properties of $E(M, K)$ are considered. Most of the properties are proved by the binary operations of sets, which is a new method in the multiobjective optimization. Then these properties are used to solve some types of multiobjective linear programming problems corresponding to Data Envelopment Analysis (DEA) models. The structures of the DEA efficient solution sets of four most representative DEA models are developed. Further more, the relationships between efficiencies of the four DEA models are deduced.

\section{Introduction and Preliminaries}

Multiobjective programming is the process of simultaneously optimizing two or more conflicting objectives subject to certain constraints (see [1-3]). Data Envelopment Analysis (DEA) is a nonparametric method in operations research and economics for the estimation of production frontiers. It is used to empirically measure productive efficiency of decision maker units $\mathrm{DMU}_{1}, \ldots, \mathrm{DMU}_{n}$ by solving the linear programming [4-6]. Charnes et al. and Wei et al. establish the equivalence of (weak) DEA efficient solutions in DEA model and (weak) Pareto solutions of multiobjective linear programming [7-9]. There is a multiobjective linear programming, corresponding to a DEA model, such that a $\mathrm{DMU}_{j_{0}}\left(1 \leq j_{0} \leq n\right)$ is (weak) DEA efficient if and only if $\left(x_{j_{0}}, y_{j_{0}}\right)$ (associating with the DMU $\left.j_{0}\right)$ is a (weak) Pareto efficient solution of the multiobjective linear programming whose feasible region is the production possibility set (see [10]).

In this paper, we propose a new representation for the set of Pareto efficient (weak efficient) points. With the help of the new representation, not only the properties of the set of Pareto efficient (weak efficient) points which are given in $[1,3,11]$ can be proved more simply, but also more new properties can be obtained. It is these new properties that reveal the relationships between the set of solutions and different multiobjective linear programmings which correspond to different DEA models. Further, the relationships between efficiencies of DMUs in different DEA models are obtained by a new way. Wei et al. [10] develop a famous method to translate production possibility sets in the intersection form and in the sum form and find all DEA efficient DMUs. For each of the four most representative DEA models, we offers a simple way to get all DEA efficient DMUs by the binary operations of sets.

Now let us recall the definition of efficiency and the representation of the set of efficient points deduced by the definition in vector optimization.

Definition 1 ([1], efficiency, weak efficiency). Given a nonempty set $M$ and a cone $K$ with int $K \neq \emptyset$ in $R^{n}, x_{0} \in M$ is called a Pareto efficient (weak efficient) point of $M$, if there is no $y \in M$ with $y \neq x_{0}$ such that $x_{0} \in y+K\left(x_{0} \in y+\operatorname{int} K\right)$. The set of all Pareto efficient (weak efficient) points of $M$ is denoted by $E(M, K)\left(E_{w}(M, K)\right)$. 
$E(M, K)\left(E_{w}(M, K)\right)$ is called the efficient (weak efficient) point set of $M$. By Definition 1, we have that

$$
\begin{array}{r}
E(M, K)=\{x \in M \mid \text { there is no } y \in M, \\
y \neq x \text { such that } x \in y+K\}, \\
\left(E_{w}(M, K)=\{x \in M \mid \text { there is no } y \in M\right. \\
\text { such that } x \in y+\text { int } K\}) .
\end{array}
$$

In [2] when $K=R_{+}^{n}$, the set is also described as follows:

$$
\begin{gathered}
E(M, K)=\{x \in M \mid M \cap(x-K)=\{x\}\}, \\
\left(E_{w}(M, K)=\{x \in M \mid M \cap(x-\text { int } K)=\emptyset\}\right) .
\end{gathered}
$$

Since $K=R_{+}^{n}$ is a convex pointed cone, (1) and (3) are equivalent. So are (2) and (4). In Section 2, we give a new representation of the efficient (weak efficient) point set, which is expressed as the difference of two sets. The idea of the new representation is motivated by the following facts in the area of DEA:

(i) the structures of the production possibility sets and the relationships between these sets (5);

(ii) the equivalence of Pareto efficiency in multiobjective linear programming and DEA efficiency in DEA model (Theorem 18);

(iii) the particularity of structures of the set of solutions to the multiobjective linear programmings corresponding to DEA models (detailed in Section 3).

For the four most representative DEA models $C^{2} R, B C^{2}$, $F G$, and $S T$ (for the details about the models, see $[4,5]$ ), each of the DEA models associates with a production possibility set which is also the feasible set of the multiobjective linear programming corresponding to this DEA model. The production possibility sets are denoted by $T_{C^{2} R}, T_{B C^{2}}, T_{F G}$, and, $T_{S T}$, respectively. The following relations hold (the structures of these production possibility sets are presented in Section 3):
(a) $T_{C^{2} R}=T_{F G} \cup T_{S T}$,
(b) $T_{B C^{2}}=T_{F G} \cap T_{S T}$,
(c) $T_{C^{2} R} \supset T_{F G} \supset T_{B C^{2}}$,
(d) $T_{C^{2} R} \supset T_{S T} \supset T_{B C^{2}}$.

It is the specialty of the relations of the production possibility sets and the equivalence of DEA efficiency and Pareto efficiency that motivate us to propose a new representation of (weak) efficient point set. Using the new representation,we obtain some new properties of efficient point set $E(M, K)$, when the set $M$ or $K$ is in form at union, intersection, sum, or difference of two sets. By these properties, it is easier to get the relationship between the DEA efficiency of the four DEA models than before.

This paper is organized as follows. Section 2 introduces the new representation of the efficient (weak efficient) point set of a set, discusses some new properties of efficient point set. Using the new expression of the set $E(M, K)\left(E_{w}(M, K)\right)$, most of these properties are proved by the binary operations of sets. The multiobjective linear programming problems corresponding to the four DEA models are studied in Section 3. The structures of the efficient point sets and the efficient solution sets of the multiobjective linear programming problems are developed, and then the relationships between DEA efficiencies of DMUs in four DEA models are revealed. Section 4 is devoted to the conclusion.

The following notations are used in the paper.

Let $M, M_{1}, M_{2}$, and $K$ be sets in $R^{n}$ :

$$
\begin{gathered}
M_{1}+M_{2}=\left\{x_{1}+x_{2} \mid x_{1} \in M_{1}, x_{2} \in M_{2}\right\}, \\
M_{1} \backslash M_{2}=\left\{x \mid x \in M_{1}, x \notin M_{2}\right\}, \\
\bar{M}=\left\{x \in R^{n} \mid x \notin M\right\}, \\
K_{0}=K \backslash\{0\} .
\end{gathered}
$$

\section{Some Properties of the Efficient Point Set}

In this section, a new representation of $E(M, K)\left(E_{w}(M, K)\right)$ is presented. Then we prove that it is equivalent to the original ones when $K$ is a cone. Lastly we focus on the properties of $E(M, K)$, when $M$ or $K$ is in the form of the union, intersection, sum, or difference of two sets. Most of the proofs are completed by the binary operations of sets, which is a new method in multiobjective optimization.

Definition 2. Given a nonempty set $M$ and a cone $K$ with int $K \neq \emptyset$ in $R^{n}$, the efficient (weak efficient) point set of $M$ with respect to $K$ is defined by

$$
\begin{gathered}
E(M, K)=M \backslash\left(M+K_{0}\right), \\
\left(E_{w}(M, K)=M \backslash(M+\text { int } K)\right) .
\end{gathered}
$$

Clearly, the following result holds.

Theorem 3. Equations (1) and (7) ((2) and (8)) are equivalent, that is,

$$
\begin{gathered}
E(M, K)=\{x \in M \text { there is no } y \in M, y \neq x \\
\text { such that } x \in y+K\}=M \backslash\left(M+K_{0}\right), \\
\left(E_{w}(M, K)=\{x \in M \mid \text { there is no } y \in M\right. \\
\text { such that } x \in y+\text { int } K\} \\
=M \backslash(M+\operatorname{int} K)) .
\end{gathered}
$$

Definition 2 gives a new representation of $E(M, K)$ that is, the efficient point set of a set $M$ is the difference between two sets. Using this new representation, we prove the properties of $E(M, K)$. Proposition 4 comes from Luc [1], Papageorgiou [3], and Guerraggio et al. [11]. We give an easier proof of this proposition.

Proposition 4. Assume that $M, M_{1}$, and $M_{2}$ are nonempty sets and $K, K_{1}$, and $K_{2}$ are cones in $R^{n}, \lambda>0$. Then

(i) $E\left(M, K_{2}\right) \subseteq E\left(M, K_{1}\right)$, if $K_{1} \subseteq K_{2}$, 
(ii) $E(\lambda M, K)=\lambda E(M, K)$,

(iii) $E\left(M_{1}+M_{2}, K\right) \subseteq E\left(M_{1}, K\right)+E\left(M_{2}, K\right)$,

(iv) $E(M+H, K)=E(M, K)$, for any $\{0\} \subseteq H \subseteq K, K$ is a convex cone.

Proof. For convenience, let $K_{10}=K_{1} \backslash\{0\}$, and let $K_{20}=$ $K_{2} \backslash\{0\}$. Since $K_{1} \subseteq K_{2}$, we have $M+K_{10} \subseteq M+K_{20}$. And then

$$
\begin{aligned}
& E\left(M, K_{2}\right)=M \backslash\left(M+K_{20}\right) \\
& \subseteq M \backslash\left(M+K_{10}\right)=E\left(M, K_{1}\right), \\
& E(\lambda M, K)=(\lambda M) \backslash\left(\lambda M+K_{0}\right) \\
&=(\lambda M) \backslash\left(\lambda M+\lambda K_{0}\right) \\
&=(\lambda M) \backslash \lambda\left(M+K_{0}\right) \\
&=\lambda\left[M \backslash\left(M+K_{0}\right)\right]=\lambda[E(M, K)] .
\end{aligned}
$$

By Theorem 3, it is sufficient to show that

$$
\begin{aligned}
& \left(M_{1}+M_{2}\right) \backslash\left(M_{1}+M_{2}+K_{0}\right) \\
& \quad \subseteq\left[M_{1} \backslash\left(M_{1}+K_{0}\right)\right]+\left[M_{2} \backslash\left(M_{2}+K_{0}\right)\right],
\end{aligned}
$$

for all $x=x_{1}+x_{2} \in\left(M_{1}+M_{2}\right) \backslash\left(M_{1}+M_{2}+K_{0}\right)$, where $x_{1} \in M_{1}, x_{2} \in M_{2}$ and $x_{1}+x_{2} \notin M_{1}+M_{2}+K_{0}$. Then $x_{1} \notin$ $M_{1}+K_{0}$, otherwise $x_{1}+x_{2} \in M_{1}+M_{2}+K_{0}$, a contradiction. Similarly $x_{2} \notin M_{2}+K_{0}$. Hence

$$
x=x_{1}+x_{2} \in\left[M_{1} \backslash\left(M_{1}+K_{0}\right)\right]+\left[M_{2} \backslash\left(M_{2}+K_{0}\right)\right] .
$$

Since $0 \in H \subseteq K$ and $K$ is a convex cone, we have $M \subset$ $M+H, H+K_{0}=K_{0}$,

$$
\begin{aligned}
E(M+H, K) & =(M+H) \backslash\left(M+H+K_{0}\right) \\
& =(M+H) \backslash\left(M+K_{0}\right) \supset M \backslash\left(M+K_{0}\right) \\
& =E(M, K) .
\end{aligned}
$$

On the other hand, For any $x \in E(M+H, K)=(M+$ $H) \backslash\left(M+K_{0}\right), x=m+h, m \in M, h \in H$. If $h \neq 0$, then $x \in\left(M+K_{0}\right)$, a contradiction. Hence, $x \in M, x \notin M+K_{0}$, that is, $x \in E(M, K)$.

A spacial case of (ii) in Proposition 4 is that

$$
E(\overbrace{M+\cdots+M}^{m}, K)=E(m M, K)=m E(M, K),
$$

if $M$ is convex and $K$ is a cone.

Besides Proposition 4, we state the following properties.

Corollary 5. Consider the following:

(v) $E\left(M, K_{1}+K_{2}\right) \subseteq E\left(M, K_{1}\right) \cap E\left(M, K_{2}\right)$,

(vi) $E\left(M, K_{1} \cap K_{2}\right) \supseteq E\left(M, K_{1}\right) \cup E\left(M, K_{2}\right)$.
Proposition 6. Let $M, G_{1}$, and $G_{2}$ be sets in $R^{n}$. Then

$$
\begin{aligned}
M \backslash & {\left[M+\left(G_{1} \cup G_{2}\right)_{0}\right] } \\
& =\left[M \backslash\left(M+G_{10}\right)\right] \cap\left[M \backslash\left(M+G_{20}\right)\right] .
\end{aligned}
$$

Proof. It is obvious that $\left(G_{1} \cup G_{2}\right)_{0}=G_{10} \cup G_{20}$ and $M+\left(G_{1} \cup\right.$ $\left.G_{2}\right)_{0}=M+\left(G_{10} \cup G_{20}\right)=\left(M+G_{10}\right) \cup\left(M+G_{20}\right)$. Hence

$$
\begin{aligned}
M \backslash & {\left[M+\left(G_{1} \cup G_{2}\right)_{0}\right] } \\
& =M \backslash\left[M+\left(G_{10} \cup G_{20}\right)\right] \\
& =M \backslash\left[\left(M+G_{10}\right) \cup\left(M+G_{20}\right)\right] \\
& =M \cap \overline{\left(M+G_{10}\right) \cup\left(M+G_{20}\right)} \\
& =\left(M \cap \overline{M+G_{10}}\right) \cap\left(M \cap \overline{M+G_{20}}\right) \\
& =\left[M \backslash\left(M+G_{10}\right)\right] \cap\left[M \backslash\left(M+G_{20}\right)\right] .
\end{aligned}
$$

Let $G_{1}=K_{1}$, and let $G_{2}=K_{2}$ in Proposition 6. We have Corollary 7.

Corollary 7. If $K_{1}$ and $K_{2}$ are cones, then

$$
E\left(M, K_{1} \cup K_{2}\right)=E\left(M, K_{1}\right) \cap E\left(M, K_{2}\right) .
$$

In the following, we investigate some new properties of the efficient set when $M$ is the union, intersection, sum, or difference of two sets.

Lemma 8. If $K$ is a convex cone, then

$$
E\left[M_{1} \backslash\left(M_{2}+K\right), K\right] \subset E\left(M_{1}, K\right) .
$$

Proof. If $E\left[M_{1} \backslash\left(M_{2}+K\right), K\right]=\emptyset$, the result obviously holds.

Otherwise, for all $x \in E\left[M_{1} \backslash\left(M_{2}+K\right), K\right]$, if $x \notin$ $E\left(M_{1}, K\right), \exists y_{1} \in M_{1}, k \in K$ such that $x=y_{1}+k, k \neq 0$, where $y_{1} \in M_{1}=\left[M_{1} \backslash\left(M_{2}+K\right)\right] \cup\left[M_{1} \cap\left(M_{2}+K\right)\right]$.

If $y_{1} \in M_{1} \backslash\left(M_{2}+K\right)$, it contradicts that $x \in E\left[M_{1} \backslash\right.$ $\left.\left(M_{2}+K\right), K\right]$. Hence $y_{1} \in M_{1} \cap\left(M_{2}+K\right) . \exists y_{2} \in M_{2}$ such that $y_{1} \in y_{2}+K$, so $x \in y_{1}+K \subset y_{2}+K \subset M_{2}+K$, which still contradicts that $x \in E\left[M_{1} \backslash\left(M_{2}+K\right), K\right]$. In consequence, $x \in E\left(M_{1}, K\right)$.

A more accurate relationship between the two efficient sets in Lemma 8 is described in the following proposition.

Proposition 9. If $K$ is a convex cone, then

$$
E\left[M_{1} \backslash\left(M_{2}+K\right), K\right]=E\left(M_{1}, K\right) \backslash\left(M_{2}+K\right) .
$$

Proof. $E\left[M_{1} \backslash\left(M_{2}+K\right), K\right] \subset E\left(M_{1}, K\right)$ obtained by Lemma 8.

Note that $\left(M_{1} \backslash M_{2}+K\right) \cap\left(M_{2}+K\right)=\emptyset, E\left[M_{1} \backslash\left(M_{2}+\right.\right.$ $K), K]=E\left[M_{1} \backslash\left(M_{2}+K\right), K\right] \cap \overline{\left(M_{2}+K\right)}$. So $E\left[M_{1} \backslash\left(M_{2}+\right.\right.$ $K), K]=E\left[M_{1} \backslash\left(M_{2}+K\right), K\right] \cap \overline{\left(M_{2}+K\right)} \subset E\left(M_{1}, K\right) \backslash\left(M_{2}+\right.$ $K)$. 
Reciprocally, for all $x \in E\left(M_{1}, K\right) \backslash\left(M_{2}+K\right), x \in$ $E\left(M_{1}, K\right), x \notin M_{2}+K$. If $x \notin E\left[M_{1} \backslash\left(M_{2}+K\right), K\right]$, $\exists y \in M_{1} \backslash\left(M_{2}+K\right)$ with $x \neq y$ such that $x \in y+K$, in contradiction to $x \in E\left(M_{1}, K\right)$. Therefore,

$$
E\left(M_{1}, K\right) \backslash\left(M_{2}+K\right) \subset E\left[M_{1} \backslash\left(M_{2}+K\right), K\right] .
$$

Usually $E\left(M_{1} \backslash M_{2}, K\right) \neq E\left(M_{1}, K\right) \backslash M_{2}$, is what Example 10 shows.

Example 10. Consider the following:

$$
\begin{gathered}
M_{1}=\left\{(x, y) \in R^{2} \mid 0 \leq x \leq 1,0 \leq y \leq 1\right\}, \\
M_{2}=\left\{(x, y) \in R^{2} \mid 0 \leq x \leq 1,0 \leq y \leq 1-x\right\}, \\
M_{1} \backslash M_{2}=\left\{(x, y) \in R^{2} \mid 0 \leq x \leq 1,1-x \leq y \leq 1\right\}, \\
E\left(M_{1}, R_{2}^{+}\right)=\{(0,0)\}, \\
E\left(M_{1} \backslash M_{2}, R_{2}^{+}\right) \\
=\{(x, y) \mid x+y=1,0 \leq x \leq 1,0 \leq y \leq 1\} .
\end{gathered}
$$

About the efficient point set of differences between two sets $M_{1}$ and $M_{2}$, Proposition 11 gives the conclusion, without requiring $K$ to be a convex but a cone.

Proposition 11. Consider the following:

$$
E\left(M_{1} \backslash M_{2}, K\right) \supseteq\left[E\left(M_{1}, K\right) \backslash M_{2}\right] \cup\left[E\left(\overline{M_{2}}, K\right) \backslash \overline{M_{1}}\right]
$$

Proof. We have

$$
\begin{aligned}
E\left(M_{1} \backslash M_{2}, K\right)= & \left(M_{1} \backslash M_{2}\right) \backslash\left[\left(M_{1} \backslash M_{2}\right)+K_{0}\right] \\
= & \left(M_{1} \cap \overline{M_{2}}\right) \cap \overline{\left(M_{1} \cap \overline{M_{2}}\right)+K_{0}} \\
\supseteq & \left(M_{1} \cap \overline{M_{2}}\right) \cap \overline{\left(M_{1}+K_{0}\right) \cap\left(\overline{M_{2}}+K_{0}\right)} \\
= & \left.\left(M_{1} \cap \overline{M_{2}}\right) \cap \overline{M_{1}+K_{0}} \cup \overline{\left(\overline{M_{2}}+K_{0}\right)}\right] \\
= & {\left[\left(M_{1} \cap \overline{M_{2}}\right) \cap\left(\overline{M_{1}+K_{0}}\right)\right] } \\
& \cup\left[\left(M_{1} \cap \overline{M_{2}}\right) \cap\left(\overline{\overline{M_{2}}}+K_{0}\right)\right] \\
= & {\left[E\left(M_{1}, K\right) \cap \overline{M_{2}}\right] \cup\left[E\left(\overline{M_{2}}, K\right) \cap M_{1}\right] } \\
= & {\left[E\left(M_{1}, K\right) \backslash M_{2}\right] \cup\left[E\left(\overline{M_{2}}, K\right) \backslash \overline{M_{1}}\right] . }
\end{aligned}
$$

Remark 12. In the previously mentioned proposition, if $\left(M_{1} \cap\right.$ $\left.\overline{M_{2}}\right)+K_{0}=\left(M_{1}+K_{0}\right) \cap\left(\overline{M_{2}}+K_{0}\right)$, the equation holds.
Propositions 13 and 15 present the properties of the efficient point sets; when $M$ is the union or intersection of two sets, respectively, $K$ is not required to be a convex.

Proposition 13. Consider the following:

$$
\begin{aligned}
E\left(M_{1} \cup M_{2}, K\right)= & {\left[E\left(M_{1}, K\right) \backslash\left(M_{2}+K_{0}\right)\right] } \\
& \cup\left[E\left(M_{2}, K\right) \backslash\left(M_{1}+K_{0}\right)\right] .
\end{aligned}
$$

Proof. We have

$$
\begin{aligned}
E\left(M_{1} \cup M_{2}, K\right)= & \left(M_{1} \cup M_{2}\right) \backslash\left[\left(M_{1} \cup M_{2}\right)+K_{0}\right] \\
= & \left(M_{1} \cup M_{2}\right) \cap \overline{\left(M_{1} \cup M_{2}\right)+K_{0}} \\
= & \left(M_{1} \cup M_{2}\right) \cap \overline{\left(M_{1}+K_{0}\right) \cup\left(M_{2}+K_{0}\right)} \\
= & \left(M_{1} \cup M_{2}\right) \cap \overline{M_{1}+K_{0}} \cap \overline{M_{2}+K_{0}} \\
= & \left(M_{1} \cap \overline{M_{1}+K_{0}} \cap \overline{M_{2}+K_{0}}\right) \\
& \cup\left(M_{2} \cap \overline{M_{2}+K_{0}} \cap \overline{M_{1}+K_{0}}\right) \\
= & {\left[E\left(M_{1}, K\right) \backslash\left(M_{2}+K_{0}\right)\right] } \\
& \cup\left[E\left(M_{2}, K\right) \backslash\left(M_{1}+K_{0}\right)\right] .
\end{aligned}
$$

Remark 14. It is obvious that if $E\left(M_{1}, K\right) \cap\left(M_{2}+K_{0}\right)=\emptyset$ and $E\left(M_{2}, K\right) \cap\left(M_{1}+K_{0}\right)=\emptyset$, then

$$
E\left(M_{1} \cup M_{2}, K\right)=E\left(M_{1}, K\right) \cup E\left(M_{2}, K\right) .
$$

\section{Proposition 15. Consider the following:}

$$
E\left(M_{1} \cap M_{2}, K\right) \supseteq\left[E\left(M_{1}, K\right) \cap M_{2}\right] \cup\left[E\left(M_{2}, K\right) \cap M_{1}\right] .
$$

Proof. Notice that $\left(M_{1} \cap M_{2}\right)+K_{0} \subseteq\left(M_{1}+K_{0}\right) \cap\left(M_{2} \cap K_{0}\right)$.

$$
\begin{aligned}
E\left(M_{1} \cap M_{2}, K\right)= & \left(M_{1} \cap M_{2}\right) \backslash\left[\left(M_{1} \cap M_{2}\right)+K_{0}\right] \\
& \supseteq\left(M_{1} \cap M_{2}\right) \backslash\left[\left(M_{1}+K_{0}\right) \cap\left(M_{2}+K_{0}\right)\right] \\
= & \left(M_{1} \cap M_{2}\right) \cap \overline{\left(M_{1}+K_{0}\right) \cap\left(M_{2}+K_{0}\right)} \\
= & \left.\left(M_{1} \cap M_{2}\right) \cap \overline{M_{1}+K_{0}} \cup \overline{M_{2}+K_{0}}\right] \\
= & {\left[\left(M_{1} \cap M_{2}\right) \cap \overline{M_{1}+K_{0}}\right] } \\
& \cup\left[\left(M_{1} \cap M_{2}\right) \cap \overline{M_{2}+K_{0}}\right] \\
= & {\left[E\left(M_{1}, K\right) \cap M_{2}\right] \cup\left[E\left(M_{2}, K\right) \cap M_{1}\right] . }
\end{aligned}
$$

Remark 16. (i) Usually the equality does not hold in Proposition 15. Let $M_{1}=\{(x, y) \mid 0 \leq x \leq y \leq 1\}$, $M_{2}=\{(x, y) \mid 0 \leq x-1 / 2 \leq y \leq 1\}$. Then

$$
\begin{gathered}
E\left(M_{1} \cap M_{2}, K\right)=\{(1 / 2,1 / 2)\}, \\
{\left[E\left(M_{1}, K\right) \cap M_{2}\right] \cup\left[E\left(M_{2}, K\right) \cap M_{1}\right]=\emptyset,}
\end{gathered}
$$


(ii) for Proposition 15, if $\left(M_{1} \cap M_{2}\right)+K_{0}=\left(M_{1}+K_{0}\right) \cap$ $\left(M_{2}+K_{0}\right)$, then

$$
E\left(M_{1} \cap M_{2}, K\right)=\left[E\left(M_{1}, K\right) \cap M_{2}\right] \cup\left[E\left(M_{2}, K\right) \cap M_{1}\right],
$$

(iii) since $M_{1} \backslash M_{2}=M_{1} \cap \overline{M_{2}}$, Proposition 11 can be obtained by Proposition 15.

\section{Efficiency of Four Most Representative DEA Models}

For each of DEA models, Charnes et al. and Wei et al. (e.g., see $[5,7,9]$ ) establish an associative multiobjective linear programming model and prove that a $\mathrm{DMU}_{j_{0}}$ is DEA efficient if and only if $\left(x_{j_{0}}, y_{j_{0}}\right)$ (associating with the $\mathrm{DMU}_{j_{0}}$ ) is a Pareto efficient solution of the multiobjective linear programming problem (for the details about DEA efficient of DMUs in DEA model, see $[4,5]$ and the references therein). This beautiful conclusion provides the multiobjective linear programming as an efficient tool to solve DEA problems. So the key point now is to find all efficient solutions of the multiobjective linear programming problems. This section offers a simple way to do this.

By the results of Section 2, in the following, we investigate the multiobjective linear programming problems corresponding to the four DEA models and develop the structures of the efficient point sets and the efficient solution sets of these programmings. Based on these, the relationships between the DEA efficiency of the four DEA models are obtained.

Denote $x_{j}=\left(x_{1 j}, \ldots, x_{m j}\right)>0\left(x_{j} \in R^{m}\right)$ to be the input vector for the $j$ th decision making unit $\left(\mathrm{DMU}_{j}\right)$, and $y_{j}=$ $\left(y_{1 j}, \ldots, y_{s j}\right)>0\left(y_{j} \in R^{s}\right)$, to be the output vector for the $j$ th decision making unit, for $j=1, \ldots, n$. For convenience the notations $x_{0}=x_{j_{0}}, y_{0}=y_{j_{0}}$, for $1 \leq j_{0} \leq n$ are given. Let $C=R_{+}^{m} \subset R^{m}$, and let $D=R_{+}^{s} \subset R^{s}$. The orderings in $R^{m}$ and $R^{s}$ are defined by $C$ and $D$, respectively. $K=\{(c, d) \mid c \in$ $C, d \in D\}, K_{1}=\{(c,-d) c \in C, d \in D\}, K_{0}=K \backslash\{0\}$, and $K_{10}=K_{1} \backslash\{0$. The production possibility set $T, T \subset\{(x, y) \mid$ $\left.x \in R^{m}, y \in R^{s}, x \geq 0, y \geq 0\right\}$, is based on postulate sets which are presented with a brief explanation (see $[4,5])$. The four most representative models are, briefly, $C^{2} R, B C^{2}, F G$, and $S T$, which correspond to different production possibility sets $T_{C^{2} R}, T_{B C^{2}}, T_{F G}$, and $T_{S T}$, respectively, [4-10]

$$
\begin{aligned}
T_{C^{2} R}= & \left\{(x, y) \mid \sum_{j=1}^{n} x_{j} \lambda_{j} \leq x,\right. \\
& \left.\sum_{j=1}^{n} y_{j} \lambda_{j} \geq y \geq 0, \lambda_{j} \geq 0, j=1, \ldots, n\right\},
\end{aligned}
$$

$$
\begin{gathered}
T_{B C^{2}}=\left\{(x, y) \mid \sum_{j=1}^{n} x_{j} \lambda_{j} \leq x, \sum_{j=1}^{n} y_{j} \lambda_{j} \geq y \geq 0,\right. \\
\left.\sum_{j=1}^{n} \lambda_{j}=1, \lambda_{j} \geq 0, j=1, \ldots, n\right\}, \\
T_{F G}=\left\{(x, y) \mid \sum_{j=1}^{n} x_{j} \lambda_{j} \leq x, \sum_{j=1}^{n} y_{j} \lambda_{j} \geq y \geq 0,\right. \\
\left.\sum_{j=1}^{n} \lambda_{j} \leq 1, \quad \lambda_{j} \geq 0, j=1, \ldots, n\right\}, \\
\left\{(x, y) \mid \sum_{j=1}^{n} x_{j} \lambda_{j} \leq x, \sum_{j=1}^{n} y_{j} \lambda_{j} \geq y \geq 0,\right. \\
\left.\sum_{j=1}^{n} \lambda_{j} \geq 1, \lambda_{j} \geq 0, j=1, \ldots, n\right\} .
\end{gathered}
$$

Obviously, the following equalities and inclusions hold:
(a) $T_{C^{2} R}=T_{F G} \cup T_{S T}$,
(b) $T_{B C^{2}}=T_{F G} \cap T_{S T}$,
(c) $T_{C^{2} R} \supseteq T_{F G} \supseteq T_{B C^{2}}$,
(d) $T_{C^{2} R} \supseteq T_{S T} \supseteq T_{B C^{2}}$.

For distinguishing the DEA efficiency of DMUs in DEA models, according to the equivalence of DEA efficiency in DEA models and Pareto efficiency in multiobjective linear programming obtained by Charnes et al. and Wei et al., we introduce the multiobjective linear programming problem corresponding to DEA models in the following. For the details about DEA models, see $[7,8]$.

Consider multiobjective linear programming problem:

$$
\begin{aligned}
& V-\min (x,-y), \\
& \text { s.t. }(x, y) \in T .
\end{aligned}
$$

For $T=T_{C^{2} R}, T_{B C^{2}}, T_{F G}$, or $T_{S T}$, the four multiobjective linear programming problems $V P_{C^{2} R}, V P_{B C^{2}}, V P_{F G}$, and $V P_{S T}$ correspond to the four DEA models respectively.

Definition 17. $\left(x_{0}, y_{0}\right)$ is called an Pareto efficient (weak efficient) solution of $(V P)$, if there is no $(x, y) \in T,(x, y) \neq$ $\left(x_{0}, y_{0}\right)$ such that

$$
\begin{gathered}
(x,-y) \leq\left(x_{0},-y_{0}\right), \\
\left((x,-y)<\left(x_{0},-y_{0}\right)\right) .
\end{gathered}
$$

Since $K$ defines the ordering in $R^{m+s}(\supset T)$, the previous inequality can be written as

$$
\begin{gathered}
\left(x_{0},-y_{0}\right) \in(x,-y)+K_{0}, \\
\left(\left(x_{0},-y_{0}\right) \in(x,-y)+\text { int } K\right) .
\end{gathered}
$$


When $K$ is a convex pointed cone, all conclusions in Section 2 hold for multiobjective programming problem $(V P)$. Theorem 18 provides the equivalency of DEA efficiency of DMUs and the Pareto efficiency of the points corresponding to the DMUs. Consequently, the structures of the sets of efficient solutions to DEA models are obtained by solving the multiobjective programmings. Then the relationships between the DEA efficiencies of DMUs in DEA models are deduced.

Theorem 18 (see [5]). A DMU ${ }_{j_{0}}$ is DEA efficient if and only if $\left(x_{0}, y_{0}\right)$ is a Pareto efficient solution of $(V P)$.

For convenience, we denote by DMU ${ }_{0} \mathrm{DMU}_{j_{0}}$. In the following, we investigate the structures of the efficient point sets and the efficient solution sets of the four vector optimization problems. Let

$$
\begin{aligned}
M_{0} & =\left\{\left(x_{j},-y_{j}\right) \mid j=1, \ldots, n\right\}, \\
T_{0} & =\left\{\left(x_{j}, y_{j}\right) \mid j=1, \ldots, n\right\}, \\
M_{C^{2} R} & =\left\{(x,-y) \mid(x, y) \in T_{C^{2} R}\right\}, \\
M_{B C^{2}} & =\left\{(x,-y) \mid(x, y) \in T_{B C^{2}}\right\}, \\
M_{F G} & =\left\{(x,-y) \mid(x, y) \in T_{F G}\right\}, \\
M_{S T} & =\left\{(x,-y) \mid(x, y) \in T_{S T}\right\} .
\end{aligned}
$$

The relations $(a),(b),(c)$, and $(d)$ imply:

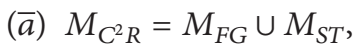

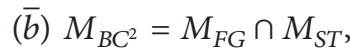

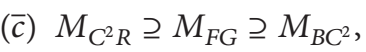

$$
\begin{aligned}
& (\bar{d}) M_{C^{2} R} \supseteq M_{S T} \supseteq M_{B C^{2}} \text {. }
\end{aligned}
$$

As an example, consider the multiobjective linear programming problem $\left(V P_{C^{2} R}\right)$. Let $E_{C^{2} R}$ denote the efficient point set, and let $S_{C^{2} R}$ denote the efficient solution set. Note that

$$
(x,-y) \leq\left(x_{0},-y_{0}\right) \Longleftrightarrow\left(x_{0},-y_{0}\right) \in(x,-y)+K .
$$

By Definitions 2 and 17,

$$
E_{C^{2} R}=M_{0} \backslash\left(M_{C^{2} R}+K_{0}\right) .
$$

Since

$$
\left(x_{0},-y_{0}\right) \in(x,-y)+K_{0} \Longleftrightarrow\left(x_{0}, y_{0}\right) \in(x, y)+K_{10},
$$

we have

$$
S_{C^{2} R}=T_{0} \backslash\left(T_{C^{2} R}+K_{10}\right) .
$$

Similar argument is applied to other three multiobjective linear programming problems. Therefore the following two theorems hold.
Theorem 19. Consider the following:

$$
\begin{aligned}
& (\overline{\bar{a}}) E_{C^{2} R}=M_{0} \backslash\left(M_{C^{2} R}+K_{0}\right) \text {, }
\end{aligned}
$$

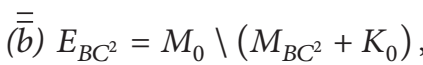

$$
\begin{aligned}
& (\overline{\bar{c}}) E_{F G}=M_{0} \backslash\left(M_{F G}+K_{0}\right) \text {, } \\
& (\overline{\bar{d}}) E_{S T}=M_{0} \backslash\left(M_{S T}+K_{0}\right) \text {. }
\end{aligned}
$$

Theorem 20. Consider the following:

$$
\begin{aligned}
& (\overline{\overline{\bar{a}}}) S_{C^{2} R}=T_{0} \backslash\left(T_{C^{2} R}+K_{10}\right), \\
& \left(\overline{\overline{\bar{b}}} S_{B C^{2}}=T_{0} \backslash\left(T_{B C^{2}}+K_{10}\right),\right. \\
& \left(\overline{\overline{\bar{c}}} S_{F G}=T_{0} \backslash\left(T_{F G}+K_{10}\right),\right. \\
& \left(\overline{\overline{\bar{d}}} S_{S T}=T_{0} \backslash\left(T_{S T}+K_{10}\right) .\right.
\end{aligned}
$$

Inclusions $(c),(d)$ and Theorem 20 imply Theorem 21.

Theorem 21. Consider the following:

$$
\begin{aligned}
& \text { (i) } S_{C^{2} R} \subseteq S_{F G} \subseteq S_{B C^{2}} \text {, } \\
& \text { (ii) } S_{C^{2} R} \subseteq S_{S T} \subseteq S_{B C^{2}} \text {. }
\end{aligned}
$$

Theorem 21 shows the relationships between the solutions of the four vector optimization problems. Therefore, by Theorem 18, the relationships between DEA efficiencies of DMUs in DEA models are as the following:

(i) if $\mathrm{DMU}_{0}$ is $C^{2} R$ DEA efficient, then it is FG DEA efficient. If $D M U_{0}$ is $F G$ DEA efficient, then it is $B^{2} C$ DEA efficient;

(ii) if $\mathrm{DMU}_{0}$ is $C^{2} R$ DEA efficient, then it is ST DEA efficient. If $D M U_{0}$ is $S T$ DEA efficient, then it is $B^{2} C$ DEA efficient.

By Proposition 13, along with the equality $(\bar{a})$ and $(\overline{\bar{a}})$, we obtain following Theorem 22 .

Theorem 22. Consider the following:

$$
\begin{aligned}
E_{C^{2} R} & =\left[M_{0} \backslash\left(M_{F G}+K_{0}\right)\right] \backslash\left(M_{S T}+K_{0}\right) \\
& =\left[M_{0} \backslash\left(M_{S T}+K_{0}\right)\right] \backslash\left(M_{F G}+K_{0}\right) \\
& =E_{F G} \backslash\left(M_{S T}+K_{0}\right)=E_{S T} \backslash\left(M_{F G}+K_{0}\right) .
\end{aligned}
$$

By Theorem 22, we conclude that if $E_{C^{2} R} \neq \phi$, then $E_{F G} \neq \phi$ and $E_{S T} \neq \phi$; by $(\overline{\bar{a}}),(\overline{\bar{c}})$, and $(\overline{\overline{\bar{d}}})$, we declaim that if $D M U_{0}$ is $C^{2} R$ DEA efficient, then it is also FG DEA efficient and $S T$ DEA efficient.

Proposition 15 and $(\bar{b})$ infer Theorem 23.

Theorem 23. Consider the following:

$$
E_{B C^{2}} \supseteq\left(E_{F G} \cap M_{S T}\right) \cup\left(E_{S T} \cap M_{F G}\right) .
$$


Consider that $E_{F G}=M_{0} \backslash\left(M_{F G}+K_{0}\right)$, and $M_{0} \subset M_{S T}$, if $E_{F G} \neq \phi$, then $E_{F G} \cap M_{S T} \neq \phi$. Similarly, $E_{S T} \cap M_{F G} \neq \phi$. Therefore, the following consequences are obtained:

(i) if $\mathrm{DMU}_{0}$ is $S T$ DEA efficient, then it is also $B C^{2}$ DEA efficient;

(ii) if $\mathrm{DMU}_{0}$ is $F G$ DEA efficient, then it is also $B C^{2}$ DEA efficient.

\section{Conclusion}

In this paper, Definition 2 presents a new representation to $E(M, K)$. Then some new properties of $E(M, K)$ are deduced by the new representation (Propositions 6 to 15 and their corollaries). Most of the properties are proved by mean of the binary operations of sets, which is a new method in multiobjective optimization. These conclusions are used to deal with the multiobjective linear programming problems corresponding to the four most representative DEA models. We investigate the structures of the efficient solution set of the four DEA models (Theorems 19 and 20) and deduce the relationships between DEA efficient solution sets of the four DEA models (Theorems 21 and 22). For each of the four DEA models, by Theorem 20, $S=T_{0} \backslash\left(T+K_{10}\right)$; that is, all the DEA efficient DMUs are obtained by the binary operations of sets. Therefore, this is a simple way to get all DEA efficient DMUs theoretically. By using the new representation, it may be able to discuss the effects upon DEA efficiency of DMUs when the number of the DMUs changes in DEA models.

\section{Acknowledgments}

This research was supported in part by the Zhejiang Natural Science Foundation of China Grant Y6110054, the Shanxi Scholarship Council of China Research Grant 2010087.

\section{References}

[1] D. T. Luc, Theory of Vector Optimization, vol. 319, Springer, Berlin, Germany, 1989.

[2] J. Jahn, Vector Optimization, Theory, Applications, and Extensions, Springer, Berlin, Germany, 2004.

[3] N. S. Papageorgiou, "Pareto efficiency in locally convex spaces. I," Numerical Functional Analysis and Optimization, vol. 8, no. 1-2, pp. 83-116, 1985.

[4] W. W. Cooper, L. M. Seiford, and K. Tone, Data Envelopment Analysis, A Comprehensive Text With Models, Applications, References and DEA-Solver Software, Springer, 2007.

[5] Q. L. Wei, Data Envelopment Analysis, Science, Beijing, Germany, 2004.

[6] A. Charnes, W. W. Cooper, and E. Rhodes, "Measuring the efficiency of decision making units," European Journal of Operational Research, vol. 2, no. 6, pp. 429-444, 1978.

[7] A. Charnes, W. W. Cooper, Q. L. Wei, and Z. M. Huang, "Cone ratio data envelopment analysis and multi-objective programming," International Journal of Systems Science, vol. 20, no. 7, pp. 1099-1118, 1989.

[8] Q. L. Wei and G. Yu, "Analyzing properties of $K$-cones in the generalized data envelopment analysis model," Journal of Econometrics, vol. 80, no. 1, pp. 63-84, 1997.
[9] Q. L. Wei, "Equivalence between weak Pareto and weak DEA efficiency in input-oriented and output-oriented DEA model," Systems Engineering-Theory \& Practice, vol. 22, pp. 72-80, 2002.

[10] Q. L. Wei, H. Yan, and G. Hao, "Characteristics and structures of weak efficient surfaces of production possibility sets," Journal of Mathematical Analysis and Applications, vol. 327, no. 2, pp. 1055-1074, 2007.

[11] A. Guerraggio, E. Molho, and A. Zaffaroni, "On the notion of proper efficiency in vector optimization," Journal of Optimization Theory and Applications, vol. 82, no. 1, pp. 1-21, 1994. 


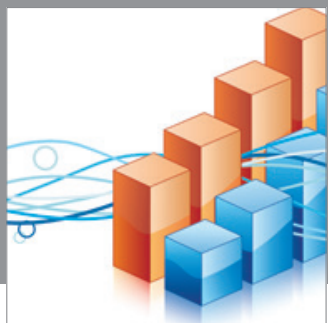

Advances in

Operations Research

mansans

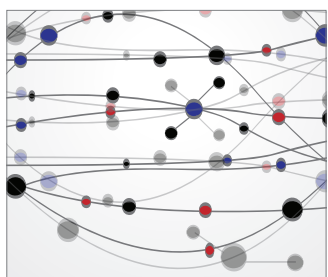

The Scientific World Journal
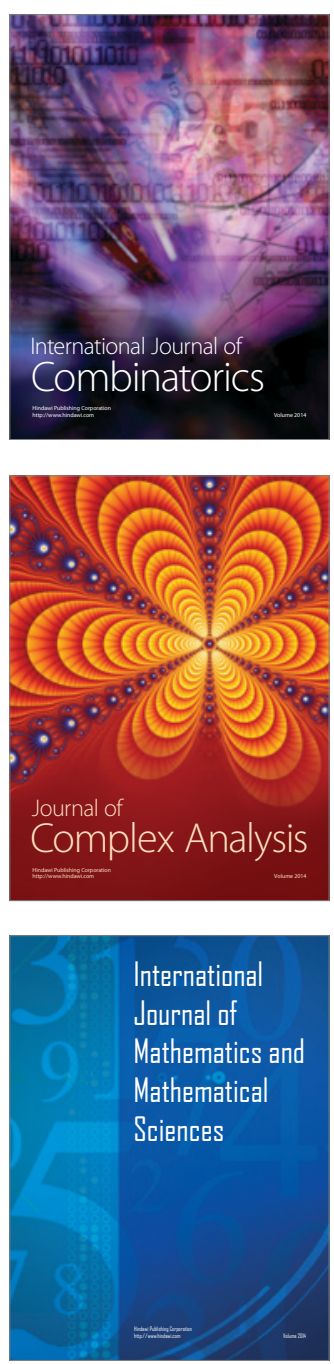
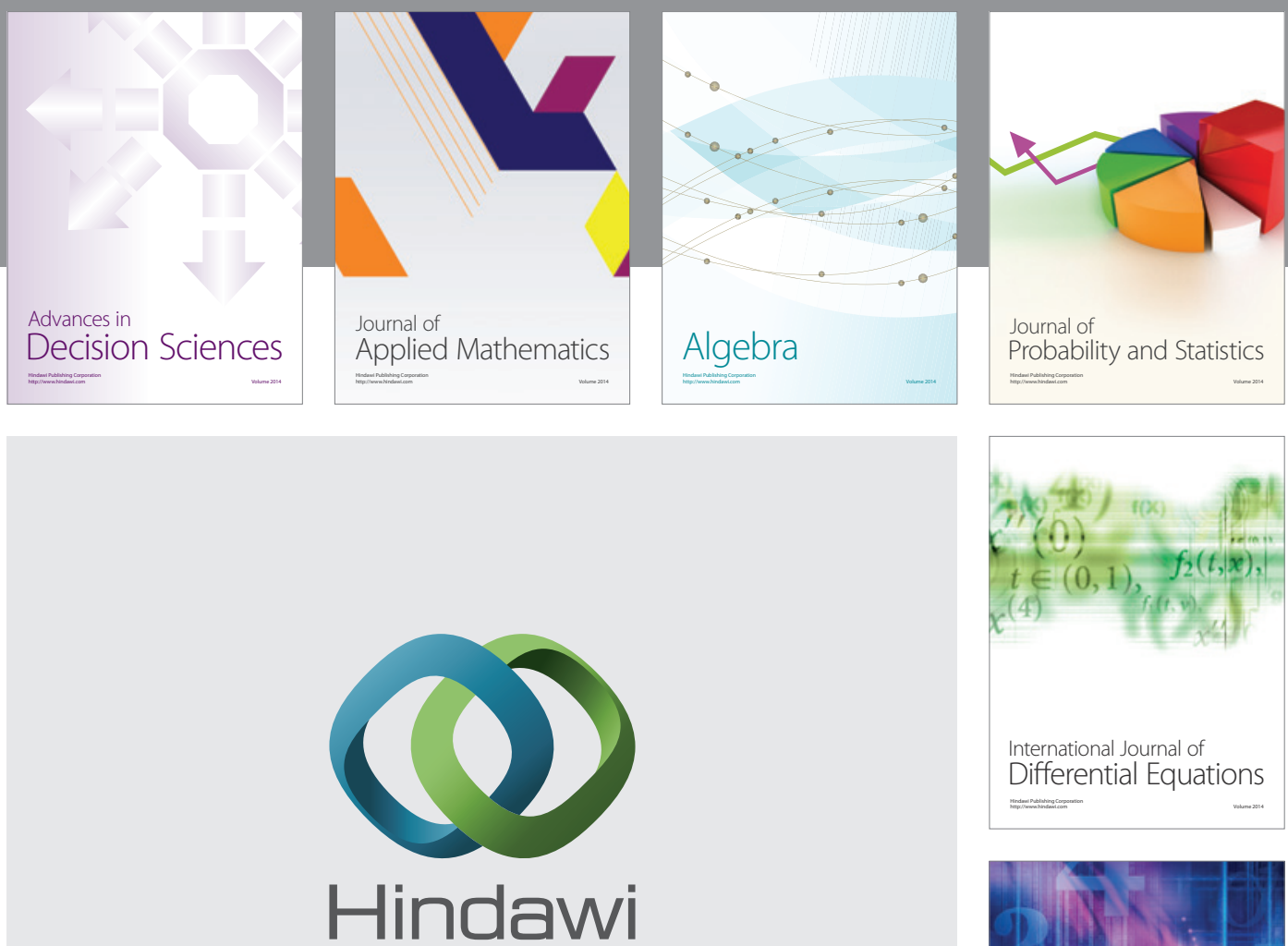

Submit your manuscripts at http://www.hindawi.com
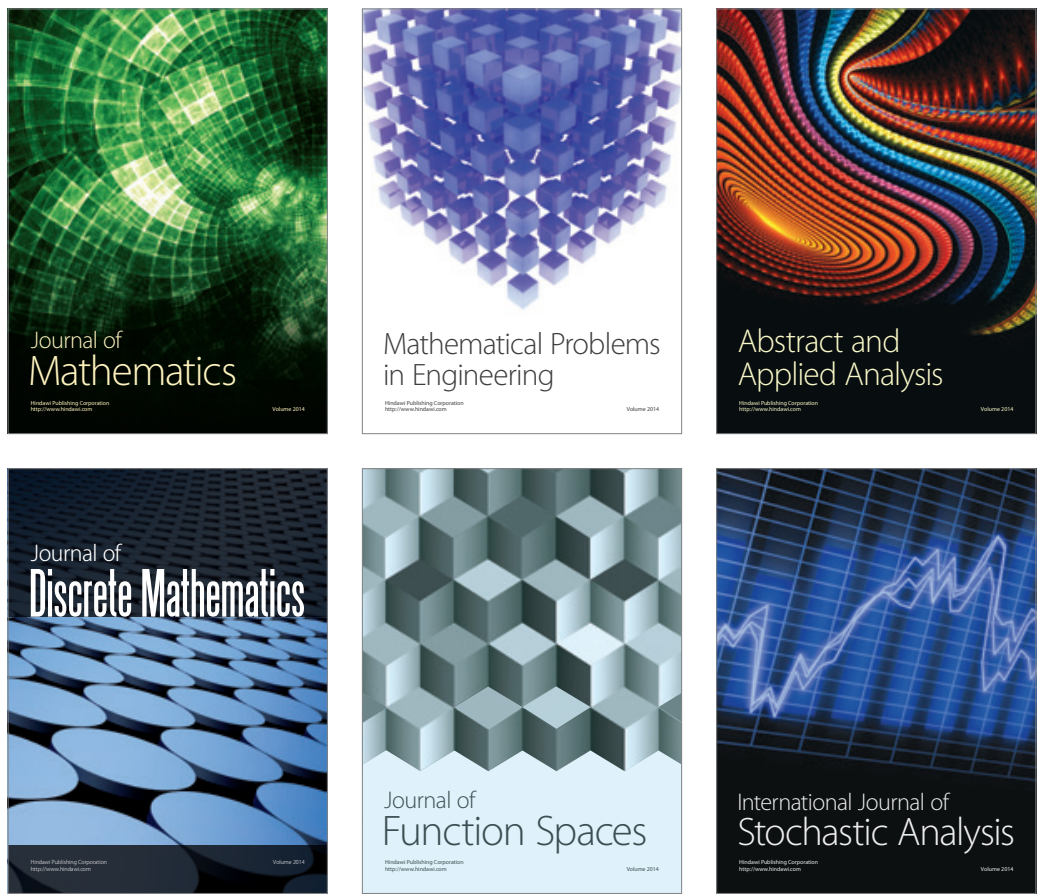

Journal of

Function Spaces

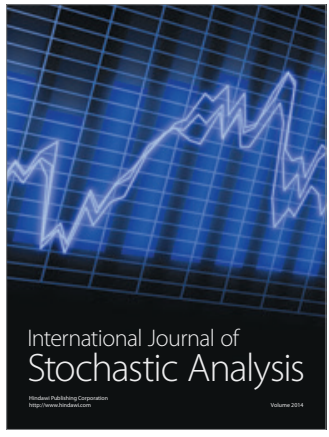

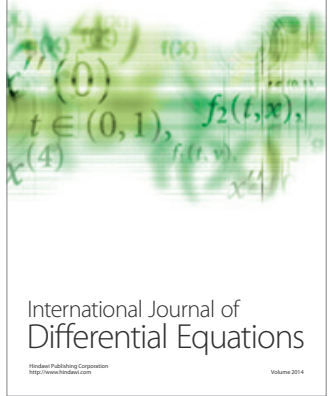
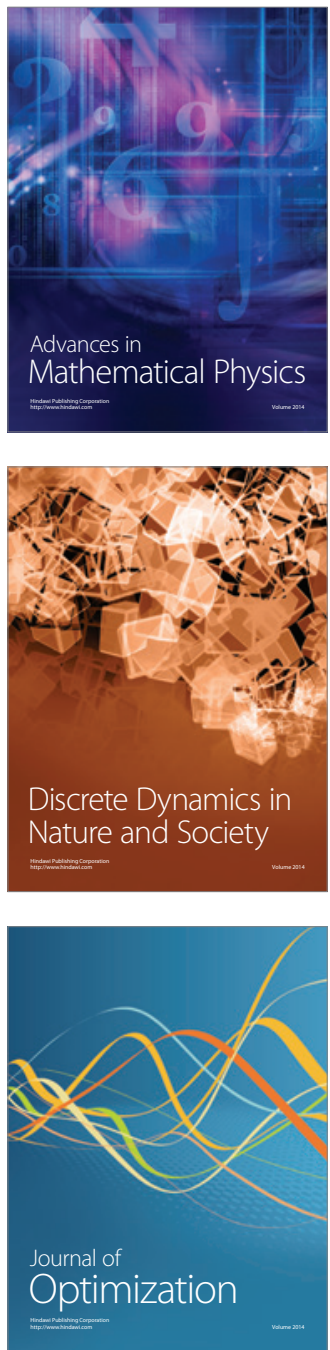